East African Medical Journal Vol. 86 No. 7 July 2009

FACTORS INFLUENCING IMMUNISATION COVERAGE IN MATHARE VALLEY, NAIROBI

L. O. Owino, MBChB, MMed (Paeds), Ministry of Health, Kisii District Hospital, P. O. Box 92, Kisii, Kenya, G. Irimu, MBChB, MMed (Paeds), Lecturer, Department of Paediatrics and Child Health, College of Health Sciences, University of Nairobi, P.O. Box 19676-00100, Nairobi, Kenya and Centre for Geographic Medicine Research, KEMRI / Wellcome Trust Research Programme, P.O. Box 43640, Nairobi, Kenya, J. Olenja, BA, MPhil, PhD, Assistant Professor, Department of Community Health and J. S. Meme, MBChB, MMed, FAAP, Dip. Med. Anthrop (Harvard), Department of Paediatrics and Child Health, College of Health Sciences, University of Nairobi, P.O. Box 19676-00100, Nairobi, Kenya

Request for reprints to: Dr. L. O. Owino, Ministry of Health, Kisii District Hospital, P.O. Box 92, Kisii, Kenya

\title{
FACTORS INFLUENCING IMMUNISATION COVERAGE IN MATHARE VALLEY, NAIROBI
}

\author{
L. O. OWINO, G. IRIMU, J. OLENJA and J. S. MEME
}

\begin{abstract}
Objective: To determine the factors that influence immunisation coverage.

Design: Cross section destrictive study.

Setting: Mathare valley slums in Central district of Nairobi, Kenya.

Subjects: Seven hundred and twelve children aged 12-23 months.

Results: Access to immunisation services was excellent at 95.6\%. However, utilisation of immunisation services was found to be suboptimal as indicated by the low fully immunised child (FIC) percentage of $69.2 \%$ and the high drop out rate between the first and third Pentavalent vaccine coverage by card or history $(\mathbf{1 2 . 0} \%)$. The immunisation status of the study population is significantly influenced by the maternal age ( $p$-value $<0.001$ ), ethnicity ( $p$-value 0.009 ) and presence of child welfare card at home ( $p$-value $<0.001$ ). Factors that contribute to the low immunisation coverage include ignorance on need for immunisations and on return dates, fear of adverse events following immunisation, negative attitude of health care providers and missed opportunities. Conclusion: The immunisation coverage in the area is low. The immunisation services are accessible but utilisation is poor.
\end{abstract}

\section{INTRODUCTION}

Immunisation has been a key component of initiatives to improve and monitor progress in child health. These include the primary health care initiative and the Millennium Development Goals (MDG). Immunisation is a key strategy and indicator of the achievement of the fourth MDG which aims at reducing the 1990's child mortality rate by two thirds by 2015 (1). UNICEF in the State of the World's Children 2006 laid emphasis and focus on excluded and invisible children (2). These include children from deprived communities discriminated against due to ethnicity, religion, HIV status, disability and children in areas of conflict. Slum children are among the most socially and economically deprived in the community and require that universal childhood immunisation services be directed to them as an entry point to provision of other essential health services as part of the actions towards including and making this vulnerable group visible.

The immunisation coverage in Nairobi province rose steadily between 2002 and 2006 with coverage of $76 \%$ in 2006 . However, the immunisation coverage in the province has not been uniform with Central district portraying problems, with both very low coverage (58\% in 2006) and poor access (Penta1 $61 \%$ in 2006) to immunisation services (source: KEPI 2006 Report). Mathare Valley is a sprawling slum in Central district of Nairobi where the immunisation services are offered in geographically accessible public health facilities. No study has been conducted in the area to determine the immunisation coverage in the area among children aged 12-23 months as recommended by the World Health Organisation (WHO). A study carried out by Kamau et al (3) to identify determinants of immunisation coverage in the area found maternal age, low level of education of mothers and lack of knowledge on vaccination as contributing to the low immunisation coverage in the area. However, this study was done in children under five years of age and the results did not thus reflect the true immunisation coverage in the area since the WHO recommends that such studies be conducted among children aged 12-23 months. Our study was conducted to determine the immunisation 
coverage and identify the factors that influence it so as to propose recommendations for interventions to increase the immunisation coverage in Mathare valley (and thus central district as a whole).

\section{MATERIALS AND METHODS}

This was a cross-sectional descriptive study that utilised both qualitative (focus group discussions and individual depthinterviews) and quantitative (cluster survey) methods of data collection. Households with children aged 12-23 months who were residents of Mathare valley for a minimum of one year were included in the study and their mothers or adult primary caretakers were interviewed. A minimum sample size of 700 was set for the study using the EPI-INFO software with immunisation coverage for the area assumed to be $65 \%$ and precision of $5 \%$ at 95\% confidence interval.

Three focus group discussions (FGDs), each consisting of six respondents, were carried out among mothers with children aged 12-23 months from different parts of the slum. Key informant interviews were carried out among traditional birth attendants, religious leaders and local village elders. In total, six key informants were recruited. The respondents were asked to list the vaccines that children should receive and their importance. Finally they were asked to discuss the reasons why some caretakers in the area do not take their children for vaccination and to suggest ways in which the immunisation services in the area could be improved.

For the cluster survey, Mathare valley was divided into 32 clusters with the assistance of the administrative Chief and village elders. We used geographical landmarks like rivers and roads and also considered the population density. Ten clusters were then selected randomly. Trained interviewers collected data from mothers or caretakers of the selected 12-23 months old children at their homes using a pre-tested structured questionnaire. In each selected cluster, the interviewers proceeded from door to door, introduced themselves and identified every household in which a 12-23 months old child resided.

Theinformation collected included demographic and socio-economic characteristics of the households, immunisation status of the child, reasons for failure to receive immunisation among those not fully immunised and caretaker's knowledge and beliefs regarding immunisation services. Information on vaccination status was collected from the child welfare card and where not available, from the respondent's recall. If the mother or caretaker was not available at the first attempted contact, a further attempt was made to interview her before leaving the cluster.
Data entry and analysis was performed using EPI INFO version 3.3.2 and SPSS 11.5 softwares (cluster survey) which were used to estimate proportions with 95\% confidence intervals and to present the results of the study graphically. Univariate and bivariate logistic regression analysis was done using immunisation status at 23 months as the outcome (dependent variable) to assess strength of association between maternal or household characteristics and immunisation status.

Complete immunisation status of children was defined using the data based on the child receiving each of the immunisations in the Kenya Expanded Programme on Immunisation (KEPI) schedule. This schedule is as follows: at birth the child should receive a dose of BCG vaccine (and birth polio vaccine); at six weeks the first polio and pentavalent (DPT-Hep $\mathrm{B}$-Hib) vaccines; at ten weeks the second polio and pentavalent vaccines; at 14 weeks the third polio and pentavalent vaccines and at nine months, the measles vaccine.

This study was approved by the Kenyatta National Hospital Research and Ethics Review Committee and informed verbal consent was sought from the respondents before the questionnaire was administered to them.

\section{RESULTS}

Focus group discussions and individual depth interviews: Participants of the FGDs generally knew the vaccines that their children should receive during infancy. The most frequently mentioned vaccines were measles and polio vaccines. The deliberations of the FGD participants on the reasons for failure to immunise children revolved around the following: caretakers' ignorance or lack of knowledge on return dates for subsequent vaccines, lack of motivation making mothers not take their children for vaccination for no apparent reason and fear of adverse events following immunisation.

A mother had this to say for failing to return her baby for subsequent vaccinations "niliogopa kurudisha mtoto wangu kwa shindano kwa sababu mtoto wangu husikia uchungu na hulia sana" which literally translated is that "I feared to return my child for the injection because they are very painful and my child cries uncontrollably."

Other factors were institutional or healthcare provider associated. These included missed opportunities to immunise eligible children resulting from poor vaccine and logistics management (stock out), missed vaccination sessions and refusal to vaccinate children born at home or whose parents never attended antenatal care clinic (ANC); negative attitude of and use of abusive language by health workers. 
A mother had this to say: "After telling the health worker that I never attended antenatal care clinic, the health worker chased me away without vaccinating my baby and told me not to come back unless I brought along the "ANC" card with me."

Another participant narrated her experience thus: "when I took my baby for vaccination, the health worker chased me away for having delivered at home. Further she made some offensive remarks about Luos and Kambas delivering in their houses like rats; I felt so humiliated and swore never to go back there".

The key informants of the individual depth interviews exhibited good knowledge of the various vaccination centres in their locality. They identified negative attitude of health workers, ignorance or lack of information by the caretakers, lack of motivation and fear of adverse events following immunisation as reasons why some children in the area were not getting vaccinations appropriately.

The cluster survey: The total number of children who were included in the survey was 712;374 (52.5\%) were males and 338 (47.5\%) females. Among these children, immunisation cards were available for $501(70.5 \%)$ while information from the other 211 children was collected from caretaker recall. The predominantethnic group among the caretakers was Luo (33.1\%) followed by Kikuyu (27.6\%). The majority of the mothers $(72.7 \%)$ had attained only primary level education while $3.8 \%$ never attended school. The source of household water supply was used as a proxy for socio-economic status. Among the study households, $74.5 \%$ drew water from commercial pay points, $24.5 \%$ had piped water within the residential plots and only $1.0 \%$ had piped water within the house.
The immunisation coverage in Mathare valley: The results show that $493(69.2 \%, 95 \% \mathrm{CI}: 66.0-73.0 \%)$ of the children were fully immunised by card and history. Only $280(39.3 \%)$ were validly fully immunised by the age of one year. The immunisation coverage by card and history was: BCG (99.2\%), Penta 1 (95.6\%), Penta $3(83.7 \%)$ and Measles $(88.3 \%)$. The lowest immunisation coverage was recorded for OPVO $(68.1 \%)$. The access to immunisation services in the area is therefore excellent at $95.6 \%$. The utilisation of immunisation services in the area is poor as evidenced by the fully immunised child (FIC) rate (69.2\%) and the Penta1-Penta3 drop-out rate of $12 \%$ (95\% CI11.3$13.7 \%$ ) (Table 1).

Factors that influence immunisation coverage in Mathare valley

Reasons why children were not immunised: Mothers whose children had not completed the scheduled immunisations in the Kenya Expanded Programme on Immunisation series were asked to state the most important reason why they had been unable to take their children for the vaccination. We got responses from 136 out of the $244(55.7 \%)$ mothers whose children had not received all the recommended vaccines. Other mothers either claimed that their children had actually received the antigensin question except that the health worker had not noted down in the card or just failed to give a response. The most commonly cited reasons were that the mother was busy, the mother was unaware of return dates for subsequent vaccine doses and that the child was sick and was therefore not taken to the health facility for immunisation (Table 2).

Table 1

Immunisation coverage status for Mathare valley as at June 2006

\begin{tabular}{|c|c|c|c|c|c|c|}
\hline Antigen & $\begin{array}{l}\text { Card } \\
\text { No. }\end{array}$ & $\begin{array}{l}\text { only } \\
(\%)\end{array}$ & $\begin{array}{r}\text { Card ar } \\
\text { No. }\end{array}$ & $\begin{array}{l}\text { history } \\
(\%)\end{array}$ & $\begin{array}{r}\text { Valid (carc } \\
\text { No. }\end{array}$ & $\begin{array}{l}\text { by } 1 \text { year }) \\
(\%)\end{array}$ \\
\hline Penta 1 & 494 & 69.4 & 681 & 95.6 & 473 & 66.4 \\
\hline Penta 2 & 482 & 67.7 & 643 & 90.3 & 445 & 62.5 \\
\hline Penta 3 & 465 & 66.2 & 596 & 83.6 & 412 & 57.9 \\
\hline Measles & 422 & 60.3 & 629 & 88.3 & 364 & 51.1 \\
\hline All doses & 400 & 56.2 & 493 & 69.2 & 280 & 39.4 \\
\hline
\end{tabular}


Table 2

Reasons why children failed to receive immunisations (cluster survey)

Reason

Frequency

$(\%)$

Ignorance or lack of information $(n=47)$

Unaware of the need of immunisation/return for subsequent doses 19

Place or time of immunisation unknown

Child was sick, not brought for immunisation

Child had already suffered from measles

Obstacles $(n=59)$

Mother busy

Family problem including sickness of mother

Time of immunisation inconvenient/Long waiting times 10

Place of immunisation too far/ lack of transport 5

Lost child welfare card

Lack of motivation $(\mathrm{n}=17)$

Forgot/Postponed the immunisation to another day

Missed opportunity $(\mathrm{n}=13)$

Child ill, taken to clinic but not immunised

8

Vaccine stock out / chased away without being vaccinated

5

Total 136

Influence of socio-demographic factors: Univariate analysis was done to identify possible predictors of immunisation status at 23 months. The following factors were significantly associated with child being fully immunised: maternal age $(\mathrm{p}=0.001)$, presence of child welfare card $(\mathrm{p} \leq 0.001)$, socio-economic status (source of household water supply) $(\mathrm{OR}=4.97$, $p=0.026)$, ethnicity $(p=0.025)$, maternal knowledge of immunisation schedule $(p=0.033)$, source of information on immunisation $(\mathrm{p}=0.033)$ and birth order $(p=0.041)$ (Table 3).

Table 3

Relationship between socio-demographic characteristics of the study population and immunisation status

\begin{tabular}{|c|c|c|c|c|c|c|c|}
\hline \multirow[t]{2}{*}{ Variable } & \multicolumn{2}{|c|}{ Frequency } & \multicolumn{2}{|l|}{ FIC } & \multirow[t]{2}{*}{$X^{2}$} & \multirow[t]{2}{*}{ OR $(95 \% \quad C I)$} & \multirow[t]{2}{*}{ P-value } \\
\hline & No. & $(\%)$ & No. & $(\%)$ & & & \\
\hline Sex of child $(n=712)$ & & & & & 2.16 & $0.79(0.57-1.10)$ & 0.141 \\
\hline Male & 374 & 52.5 & 268 & 71.7 & & & \\
\hline Female & 338 & 47.5 & 225 & 66.6 & & & \\
\hline Birth order $(n=685)$ & & & & & 8.25 & & 0.041 \\
\hline 1 & 251 & 36.6 & 173 & 68.9 & & & \\
\hline 2 & 199 & 29.1 & 133 & 66.8 & & & \\
\hline 3 & 124 & 18.1 & 96 & 77.4 & & & \\
\hline $4>$ & 111 & 16.2 & 67 & 60.4 & & & \\
\hline
\end{tabular}




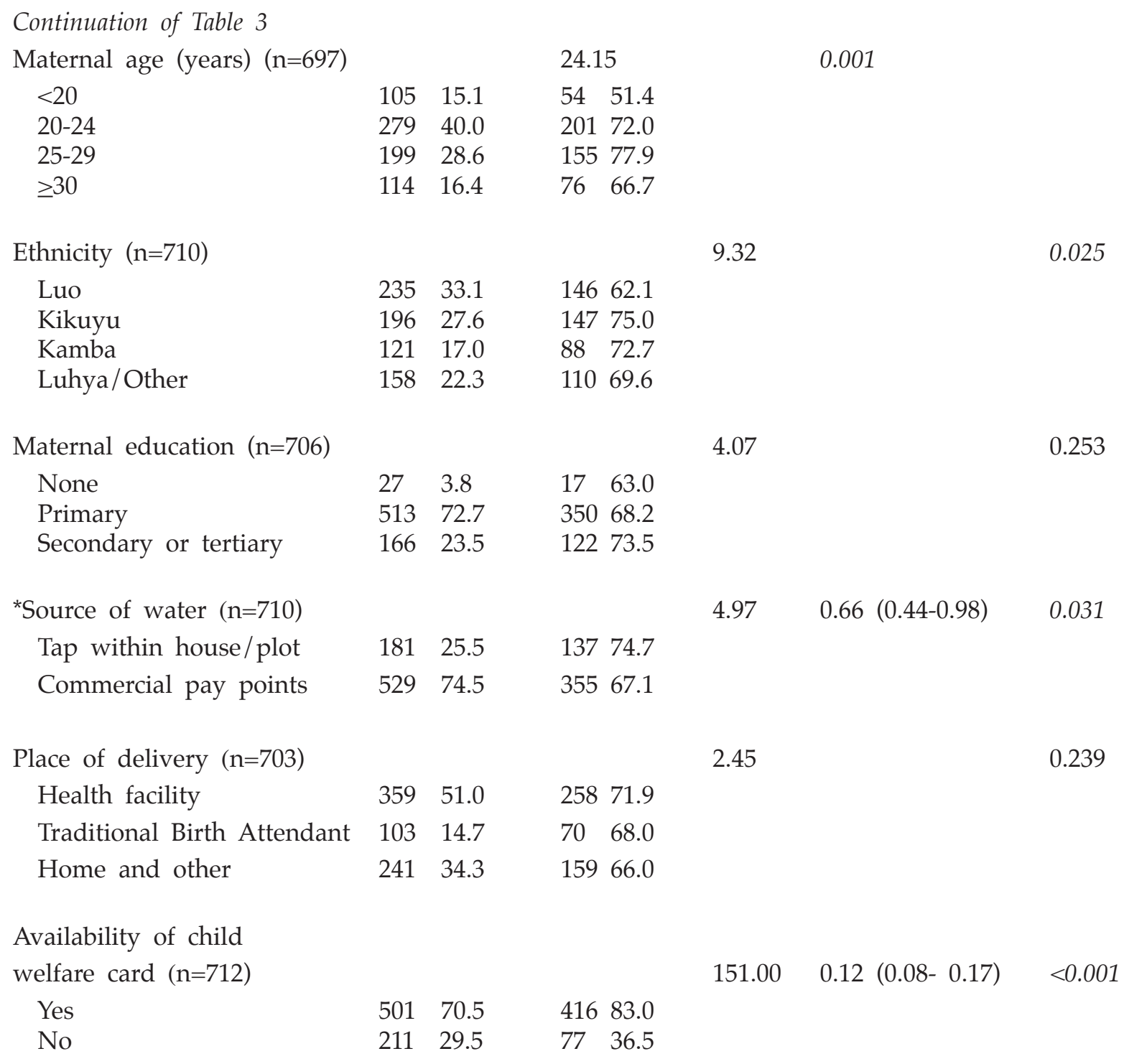

\footnotetext{
* Source of household water supply used as a proxy for socio-economic status; those with piped water within plot or within house were grouped in the higher SES and those who draw water from a commercial pay point grouped in the low SES group.

FIC=Fully Immunised Child
}

Bivariate logistic regression analysis was done using immunisation status at 23 months as the outcome (dependent variable) to assess strength of association between immunisation status and maternal and household characteristics. The factors included in the model were: ethnic groups, maternal age, presence of child welfare card, birth order and socio-economic status (SES). Among these factors, ethnic group ( $p=$ $0.006)$, maternal age $(p=0.05)$ and presence of child welfare card $(\mathrm{p}<0.001)$ were found to be significantly associated with immunisation status among the study population. Luo children were more likely to be incompletely immunised compared to children of other ethnic groups (OR 1.6, 95\% C.I 1,20-2.30); Children of teenage mothers were two and a half times more likely to be incompletely immunised compared to children born to older mothers (OR 2.5,
95\% CI 1.56-3.81) while children who did not have a child welfare card (CWC) were eight and a half times more likely to be incompletely immunised compared to those who had a CWC (OR 8.52, C.I 5.82-12.48).

\section{DISCUSSION}

The Kenya Expanded Programme on Immunisation (KEPI) was set up in 1980 with the mandate to provide childhood immunisation to all children in Kenya in accordance with the WHO/EPI guidelines. In line with the Global Immunisation Vision and Strategy (2006-2015) (1), KEPI has adopted the 90/80 goal which aims at ensuring that immunisation coverage level of $90 \%$ is attained country wide with a minimum of $80 \%$ coverage in all districts with all vaccines by 2010. 
Access to immunisation services in Mathare valley as evidenced by the crude Penta I coverage is excellent at $95.6 \%$. There is however suboptimal utilisation of immunisation services as evidenced by the low percentage $(69.2 \%)$ of fully immunised child (FIC) and the high Penta1-Penta3 drop-out rate (12\%). According to the WHOrecommendations, a drop-out rate of less than $10 \%$ denotes good performance; rates greater than $10 \%$ denote problems with continuity and quality of services (4).

Socio-demographic factors that were associated with failure to complete the vaccination schedule included young maternal age. Luo ethnicity and absence of the child welfare card (CWC). Only 70.5\% of the children had a CWC at the time of the survey. This is of significance, since as in other studies $(5,6)$, it was found that children who did not have CWCs were more likely to be incompletely immunised compared to children whose CWCs were available. In this community, mothers were less likely to take their children for vaccination for fear of being chased away or humiliated by the health care providers if they did not have a CWC. Further it emerged that most mothers donot know theimmunisation schedule and depend on the health workers to write for them the return date for subsequent immunisations on the CWC. Thus those who do not have CWCs are likely to fail to realise when the next vaccines are due and may fail completely to take their children for these immunisations. Ignorance on aspects of immunisation such as return dates accounted for $34.6 \%$ of all the children who failed to complete the vaccination schedule.

The maternal age was significantly related to immunisation status of the children. Children born to teenage mothers were found to have 2.5 times increased likelihood of being incompletely immunised compared to children of older mothers. Other investigators have also found teenage motherhood to have a negative influence on the likelihood of completing immunisation schedule $(3,6)$. In this study setting, teenage mothers were less likely to utilise health care services too. For example, teenage mothers were more likely to deliver outside a health facility compared to other mothers.

The children born to Luo mothers were 1.6 times more likely to beincompletely immunised compared to children born to mothers from other ethnic groups. Luo ethnic group appears to be the dominant group in the study area with $33.1 \%$ of all the respondents belonging to this ethnic group. Other authors have also identified Luo ethnicity to be a significant risk factor for failure to complete the vaccination schedule $(8,9)$.

Reasons why the Luo as a group appear less likely to utilise immunisation services are not understood. However, in the study area, Luo children belonged to households of lower socio-economic status compared to other households; Luo mothers were of lower educational level; and they were more likely to deliver outside a health facility. Low maternal educational level and low socio-economic status have been demonstrated to have adverse influence on immunisation coverage (7). Reasons why Luo ethnicity seems to be a risk factor for under immunisation needs to be investigated further so as to inform appropriate interventions to improve immunisation service utilisation in areas populated by this ethnic group.

Health system factors and vaccination status: Specific behaviours of the health workers such as rudeness and insensitivity such as was evident in the area, deter care givers, who feel disparaged and therefore become less motivated to return to health facilities to complete the vaccination schedule (10). Missed opportunities to immunise were responsible for failure to receive vaccination by $9.5 \%$ of the children who did not complete their vaccinations though this is lower than that found by other workers in similar settings $(11,12)$. There is however still a need to work towards eliminating the cases of missed opportunity in the facilities serving Mathare valley as a strategy for increasing the immunisation coverage in the area.

Fear of adverse effects following immunisation (AEFls) was identified to be playing a role in contributing to the high drop-out rates. Fear of adverse effects has also been found to contribute to failure to receive vaccination by other authors $(10,13)$. Thus, adequate provision for communication strategy must be put in place to address ignorance by caretakers on the importance of immunisation and to address concerns such as AEFls, rumours and other prohibitive religious and cultural practices among members of the community. In addition health education talks should include management of minor AEFls such as pain and fever with simple remedies at home.

Unpleasant immunisation operating procedures such as stripping children for long on the queues while waiting to be weighed, long queues, lateness and absenteeism of health workers (missed vaccination sessions), fee charges for vaccination services and vaccine supplies stockouts were also identified as obstacles to immunisation service utilisation in the area. Thus there is need for supportive supervision to the facilities to provide opportunities for on the job training to deal with the above obstacles and to improve satisfaction and compliance with immunisation appointments.

The District Health Management Teams that serve Mathare valley should put in place an appropriate social mobilisation and communication strategy to increase awareness and utilisation of the immunisation services in the area. Mothers also ought to be taught that minor illnesses are not a contraindication for immunisation and should not 
miss immunisation sessions because of illness of the child. In addition, a mechanism for defaulter tracing should be put in place to reduce on the rate of drop out.

Among the study limitations was that some mothers whose children did not complete the vaccination schedule did not give any reason for that and this could have introduced some bias in the analysis of the reasons for failure to complete the vaccination schedule for the children. Some mothers (29.5\% of those recruited into the household cluster survey) did not have a child welfare card of their children during the study. In these circumstances, information on vaccination status was collected from history (parental recall) alone. This reduces the reliability of data collected in this manner.

\section{ACKNOWLEDGEMENTS}

This study was made possible through financial support from the World Health Organisation (WHO) Kenya country office. We are also indebted to the Kenya Expanded Programme on Immunisation manager, Dr. Tatu Kamau for recommending the study for funding. Thanks also go to the members of Pride Mathare Self Help group for volunteering their time and energy as data collectors and to the mothers of Mathare valley and the other respondents that provided the information to make this study a success.

\section{REFERENCES}

1. WHO Department of Immunisation, Vaccines and Biologicals, 2005. Global Immunisation Vision and Strategy framework 2006-2015.

2. UNICEF, The state of the world's children 2006. December 2005.
3. Kamau, N. and Esamai, F.O. Determinants of immunisation coverage among children in Mathare Valley. East Afr. Med. J. 2001; 178: 590-594.

4. $\mathrm{MOH}, \mathrm{Kenya}$ Expanded Program on Immunisation. Performance monitoring handbook. 2003; Chapter 4: 27.

5. Ardythe, L.M., Rosenthal, I., Hassan, D. L. et al. Population based study of access to immunisation among Virginia children served by public, private and military health care system. Pediatrics. 1998; 101: $1-10$.

6. Sonoiya, S.D. Factors influencing measles immunisation in children of an urban slum. Post graduate Desertation, University of Nairobi, 1993.

7. Hugh, R.W., Leanne. D., Tegang, S.P. et al. Coverage and costs of childhood vaccination in Cameroon. Bull. WHO. 2004; 84.

8. CentralBureau of statistics, Kenya.KenyaDemographic Health Survey (KDHS), 2003; Pp 136-140.

9. Bjerregaard, P. and Mutie, D.M. Immunisation coverage in Kenya 1987. East Afr. Med. J. 1988; 65: 811-819.

10. Waisbord, S. and Larson, H. Why invest in communication for immunisation: evidence and lessons learned. A joint publication of the health communication partnership of John Hopkins School of public health and UNICEF. 2005.

11. Borus, P.K. Missed opportunities and inappropriately given vaccines reduce immunisation coverage in facilities that serve slum areas of Nairobi. East Afr. Med. J. 2004; 81: 124-129.

12. Tugumusirize, F., Tumwine, I.K. and Mworozi, E. Missed opportunities and caretaker constraints to childhood vaccinations in a rural area in Uganda. East Afr. Med. J. 2002; 79: 347-354.

13. Matsumura, T., Nakayama, T. Okamoto, S. et al. Measles vaccine coverage and factors related to uncompleted vaccination among 18-month old and 36-month old children in Kyoto, Japan. BMC Public Health. 2005; 5: 59. 